\title{
Low skeletal muscle mass predicts frailty in elderly head and neck cancer patients
}

\author{
C. D. A. Meerkerk ${ }^{1}$ N. Chargi ${ }^{1}$ P. A. de Jong ${ }^{2} \cdot$ F. van den Bos $^{3} \cdot$ R. de Bree ${ }^{1,4}$
}

Received: 20 January 2021 / Accepted: 19 April 2021 / Published online: 6 May 2021

(c) The Author(s) 2021

\begin{abstract}
Purpose Treatment of head and neck cancer (HNC) carries a high risk of adverse outcomes in patients, especially in frail elderly. Therefore, it is important to identify patients in which treatment benefits outweigh the risk of any adverse outcome. Although the comprehensive geriatric assessment (CGA) identifies frailty, it is a time-consuming tool. Instead, measurement of skeletal muscle mass and strength (sarcopenia) may be a promising and time-efficient biomarker for frailty. The aim of this study was to examine the association between sarcopenia and frailty assessment tools, such as the CGA, Fried criteria and the Groningen Frailty Indicator (GFI).

Methods A retrospective study was performed in elderly patients ( $\geq 70$-years) with HNC. Sarcopenia was defined as the combination of reduced handgrip strength (HGS) and low skeletal muscle mass (SMM), according to the EWGSOP-2 criteria. SMM was measured on routinely available diagnostic imaging and corrected height: skeletal muscle index (SMI). A CGA was performed by a geriatrician. Frailty screening was performed using the GFI and the Fried criteria.

Results In total, 73 patients were included of which 33 were men (45.2\%) and 40 women (54.8\%). Frail patients diagnosed by CGA were more likely to have low SMI, sarcopenia, more comorbidities and were at high risk for malnutrition (all $p<0.05$ ). In multivariate regression analysis, the only significant predictor for frailty diagnosed by CGA was SMI (OR $0.9, p<0.01)$ independent of comorbidity and muscle strength.

Conclusion Low SMI and sarcopenia are associated with frailty in elderly HNC patients. Low SMI predicts frailty and is a promising time-efficient and routinely available tool for clinical practice.
\end{abstract}

Keywords Sarcopenia $\cdot$ Comprehensive Geriatric Assessment $\cdot$ Muscle function $\cdot$ Handgrip strength $\cdot$ Skeletal muscle index

R. de Bree

r.debree@umcutrecht.nl

C. D. A. Meerkerk

c.d.a.meerkerk@students.uu.nl

N. Chargi

n.chargi@umcutrecht.nl

P. A. de Jong

p.deJong-8@umcutrecht.nl

F. van den Bos

f.vandenBos@umcutrecht.nl

1 Department of Head and Neck Surgical Oncology, Division

of Imaging and Oncology Center, University Medical

Center Utrecht, Heidelberglaan 100, 3584, CX, Utrecht,

The Netherlands
2 Department of Radiology. Division of Imaging and Oncology, University Medical Center Utrecht and Utrecht University, Heidelberglaan 100, 3584, CX, Utrecht, The Netherlands

3 Department of Geriatric Medicine, University Medical Center Utrecht, Heidelberglaan 100, 3584, CX, Utrecht, The Netherlands

4 Department of Head and Neck Surgical Oncology, University Medical Center Utrecht, House Postal Number Q.05.4.300, PO BOX 85500, 3508, GA, Utrecht, The Netherlands 


\section{Introduction}

Head and neck cancer (HNC) is among the most frequent malignant tumors in the world with an annual incidence of more than 650,000 cases and 330,000 deaths [1]. Of these patients, more than $60 \%$ have an age at diagnosis of 60 years or more [2]. With the global aging of the worldwide population, it is to be expected that the incidence of HNCs will increase. Besides advanced age, a significant amount of pre-existent comorbidities in HNCs patients is an additional negative prognostic factor that reduces overall survival [3].

Treatment of HNCs is often complex and requires, based on tumor-specific and patient-specific characteristics, surgery with or without adjuvant (chemo)radiotherapy or radiotherapy with or without chemotherapy with salvage surgery in reserve for residual or recurrent loco regional disease [4]. These treatments are effective, but have significant risk of toxicities, complications, and even mortality [5]. Treatment could also decrease quality of life, for instance speech problems, fatigue or trouble with social eating caused by dry mouth, and swallowing problems $[6,7]$.

Due to the growing incidence of both HNCs worldwide and the global aging of the population, it is of great importance to identify key predictive and prognostic factors for treatment outcomes in older patients with HNC. This knowledge can be useful for clinicians and patients in (shared) decision-making weighing suitability of treatment, prognosis, and expected quality of life. Although this knowledge is also important in younger HNC patients, it is even more warranted in older HNC patients due to their vulnerability, decreased physical and mental compensation mechanisms compared to younger patients. This vulnerability is also being referred to as frailty.

A comprehensive geriatric assessment (CGA) is the most appropriate way to detect frailty [8]. A CGA is a multidisciplinary, multidimensional, and systematic assessment, and consists of validated scales to identify impairments in the four geriatric domains: somatic, functional, nutritional, and psychosocial [8]. Frailty is associated with poor treatment outcomes and health-related quality of life [7]. Because performing CGA is time-consuming and not all patients will benefit from a CGA, screening methods have been developed to identify those at risk for adverse health outcomes and who may benefit from a CGA. However, the available frailty screening methods may have insufficient discriminative power to select patients for further assessment [9].

Sarcopenia also frequently observed in older patients is suggested as a more reliable, inexpensive and easy alternative for frailty screening questionnaires in $\mathrm{HNC}$ patients [10]. However, there is much discussion on different definitions of frailty and sarcopenia [11]. By the European Working Group on Sarcopenia in Older People (EWGSOP-2), sarcopenia is described as a generalized and progressive loss of muscle function (MF) and skeletal muscle mass (SMM), caused by adverse muscle changes that accrue across a lifetime [12]. Sarcopenia itself is also related with adverse health outcome, such as chemotherapy dose-limiting toxicity [13], increased incidence of postoperative complications, and decreased survival [14, 15].

The relation between low skeletal muscle mass, measured using CT of the head and neck, and frailty screening methods was recently reported by Zwart et al. [10]. However, the direct relation of sarcopenia and CGA, as gold standard for frailty, has yet to be determined.

Therefore, the aim of this study was to examine the association between sarcopenia, defined as the combination of low muscle strength and low muscle mass, and frailty, diagnosed by CGA. Our secondary aim was to examine the association between sarcopenia and the frailty Fried criteria and the Groningen Frailty Indicator (GFI) frailty screening test.

\section{Materials and methods}

\section{Ethical approval}

The design of this study was approved by the Medical Ethical Research Committee of the University Medical Center Utrecht (approval ID 17-365/C). All procedures in this study were in accordance with the ethical standards of the institutional and/or national research committee and with the 1964 Helsinki declaration (Version 2008) and its later amendments or comparable ethical standards. All data were handled according to general data protection regulation (GDPR).

\section{Patients and study design}

This study was designed as a single-center retrospective study. Older patients ( $\geq 70$ years old) with pathologically proven head and neck squamous cell carcinoma (HNSCC) treated between April 2015 and February 2018 of whom results of a CGA, Fried Frailty criteria, GFI screening questionnaire, and pre-treatment CT or MRI during their diagnostic work-up were available, were included in this study. In this period, at our center, elderly HNSCC patients were offered geriatric assessment, but patients could refuse. As a consequence, not all older patients underwent frailty assessment at that time. Histologic tumor types other than squamous cell carcinoma were excluded. 
Relevant demographic and clinical variables were collected from patient's medical record: age at diagnosis, sex, body mass index (BMI), percentage of weight loss in 6 months prior to diagnosis, smoking status, alcohol use, nutritional status at diagnosis as evaluated by the Malnutrition Universal Screening Tool (MUST), comorbidities as evaluated by the Charlson comorbidity index (CCI), localization of the tumor, tumor type (primary, second primary or recurrence), and the TNM stage according the 8th edition of the UICC tumor classification of malignant tumors.

\section{Sarcopenia}

\section{Definition of sarcopenia}

Sarcopenia was defined as the combination of low MF, as determined by muscle strength, and low muscle quantity, as determined by SMM, according to the recommendation by the EWGSOP-2 and further explained below [12].

\section{Muscle function: muscle strength}

Overall muscle strength is strongly related with isometric handgrip strength (HGS) [16]. HGS was measured using a Jamar hydraulic handheld dynamometer according to the recommendations of the American society of hand therapist's (ASHT) and expressed in kilograms (kg). Patients were asked to squeeze maximally with each hand. The average score of the left and right hands was used for analysis. Patients had low HGS if the mean HGS was below $27 \mathrm{~kg}$ (men) or below $16 \mathrm{~kg}$ (women) [12].

\section{Skeletal muscle mass}

SMM was measured as cross-sectional muscle area (CSMA) on pretreatment CT or MRI imaging of the head-and-neck area at the level of the third cervical vertebrae (C3). The axial slide of the imaging which showed both transverse processes and the entire vertebral arc was selected for the segmentation of muscle tissue. For CT imaging, muscle area was defined as the pixel area between the radio density range of -29 and +150 Hounsfield units (HU), which is specific for muscle tissue [17]. For MRI, muscle area was manually segmented, and fatty tissue was manually excluded (Fig. 1).

Segmentation of muscle tissue was manually performed using the commercially available software package SliceOmatic (version 5.0, Tomovision, Canada) by a single researcher (C.M.) who was blinded for outcome regarding frailty and sarcopenia. Cross-sectional muscle area at the level of C3 was converted to CSMA at the level of L3 using a previously published formula [18]. The lumbar skeletal muscle index (SMI) was calculated by correcting SMM at the level of L3 for height. Patients had a low SMI if this value was below $43.2 \mathrm{~cm}^{2} / \mathrm{m}^{2}$; this cut-off value was established in a separate cohort of patients with head-and-neck cancer [13].

\section{Comprehensive geriatric assessment (CGA)}

The CGA conducted in this study consists of four domains; the somatic, psychological, functional and social domains and was performed by a geriatrician. Specific, validated tools per geriatric domain were used. For the somatic domain, the Charlson Comorbidity Index (CCI)[19], the Malnutrition Universal Screening Tool (MUST) [20], and polypharmacy are used. The psychological domain was examined by the Mini Mental State Examination (MMSE) [21] for cognitive function and Geriatric Depression Scale (GDS) [22] for depression. For the functional domain, activities of daily living (ADL) were examined with The Katz Activities of Daily Living (KATZ-6) [23] and KATZ-9 was used for scoring Instrumental ADL [24]. Social status was determent on questions about current living situation, social activities, presence of informal care system/social support. Each instrument was defined as abnormal according to validated cut-off scores. The cut-off scores are listed in Table 1.
Fig. 1 Example of segmentation of skeletal muscle tissue at the level of the third cervical vertebra (C3). Two identical axial contrast enhanced computed tomography (CT) slides at the level of $\mathrm{C} 3$; left shows the muscle tissue unsegmented, right shows both sternocleidomastoid and paravertebral muscles segmented in red

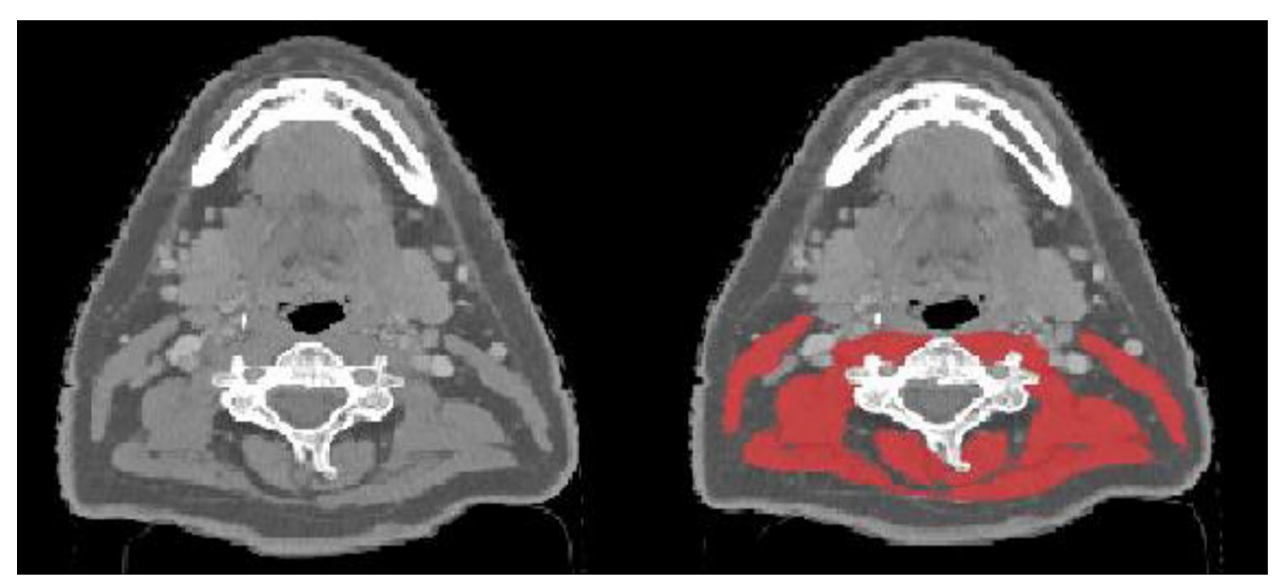


Table 1 Overview of the selected screening instruments for CGA

\begin{tabular}{lllc}
\hline Geriatric domain & & Measure & Score range or (cut-off) \\
\hline Somatic & Comorbidity & CCI & $0-31$ \\
& Nutrition & MUST & $0-3(\geq 2)$ \\
& Medication & - & Ordinal $(>4)$ \\
Psychological & Cognition & MMSE & $0-30(\leq 24)$ \\
& Depression & $G D S-2$ or & $0-2(\geq 1)$ \\
& & $G D S$ 15 & $0-15(\geq 6)$ \\
Functional & Function & ADL KATZ & $0-6,(\geq 1)$ \\
& & IADL KATZ & $0-9,(\geq 1)$ \\
Social & & Living situation, social activities & $0-3(\geq 2)$ \\
& & and informal care system & \\
\hline
\end{tabular}

$A D L$ activities of daily living, IADL instrumental activities of daily living, MMSE mini-mental state exam, GDS geriatric depression scale, MUST malnutrition universal screening tool, CCI charlson comorbidity index
Overall, a patient was considered frail if the CGA had an abnormal outcome on at least two of the instruments used.

\section{Fried frailty criteria}

The Fried Frailty criterion is an operational definition of physical frailty based on the presence of three or more of the following five criteria: unintentional weight loss, exhau stion, low physical activity level, slow gait speed, and low handgrip strength [25]. In older patients with cancer, the sensitivity and specificity of the Fried frailty criteria for predicting frailty, based on CGA, are amongst 25-37\% and $86-96 \%$, respectively [9]. The Fried frailty criteria are known to be useful in predicting complications, length of hospital stay and other adverse health outcome in patients with HNC [26].

\section{Groningen frailty indicator}

GFI is a 15-item frailty screening tool to evaluate frailty status in geriatrics through loss of function and resources in physical, social, and psychological domains. Patients were categorized as non-frail (GFI $<4)$ and frail $(\mathrm{GFI} \geq 4)$ [27]. In older patients with cancer the sensitivity and specificity of the GFI for predicting frailty, based on CGA, are amongst 39-62\% and 69-87\%, respectively [9]. The GFI is also useful in predicting postoperative complications, however, this questionnaire is not specially designed for oncological patients [26].

\section{Statistical analysis}

Data analyses were performed using IBM SPSS statistics 25. First, the patient cohort was described regarding the baseline. Continuous data are represented as mean \pm standard deviation (SD). Categorical data are represented as a number and percentage of total.
MF was presented dichotomously as low MF and normal MF based on previously published gender specific cut-offs for HGS. The SMM, was presented dichotomously as low SMI and normal SMI based on previously published specific cut-offs for SMI. Sarcopenia was presented dichotomously as sarcopenic (only if patients had a low HGS and low SMI) and non-sarcopenic (all other patients).

Frailty was presented dichotomously as frail and non-frail based on abovementioned and previously published cut-offs for frailty based on the CGA, Fried criteria or GFI. Independent sample t tests or Chi-square statistics were used for analyzing differences between the frequencies of each categorical variable with the presence or absence of sarcopenia and presence or absence of frailty.

Univariate logistic regression analyses were performed, with sarcopenia or frailty as dependent variable and the baseline variables as independent variables. Variables were selected based on clinical relevance by exploring literature. Variables that were statistically significant $(\alpha<0.05)$ in the univariate regression were included in the multivariate logistic regression. In this way, odds ratios (ORs) and 95\% CIs were provided.

\section{Results}

In total, 85 patients were referred to the geriatrician in the inclusion period. Because 12 patients did not undergo full CGA or Fried Frailty criteria or GFI screening, questionnaires were not complete, finally 73 patients were included. The mean age was 81.73 (6.24 SD). The majority of the patients was female $(55 \%)$. The mean BMI was 26.80 (5.70 SD) and most of the patients did not report loss of weight 6 months prior to diagnosis (63\%). The majority of the patients used alcohol $(56 \%)$ and were former smokers (55\%). Most patients had a high CCI comorbidity score 
of $>6(63 \%)$. According to the TNM classification, most patients had stage IV disease (44\%).

Of the included 73 patients, 33 (45\%) patients had low muscle strength, 58 (79\%) had low SMI. A total of 24 (33\%) patients were defined as sarcopenic. Based on the CGA, 39 (54\%) patients were defined as frail. Based on the frailty Fried criteria, 21 (29\%) patients were defined as frail, as the GFI defined 38 (52\%) patients frail. An overview of the characteristics of patients is listed in Table 2.

\section{Correlations}

Table 2 shows statistically significant differences in CGA, Fried criteria, age at diagnosis, and BMI between patients with and without sarcopenia. Patients with sarcopenia were more likely to be frail according to the CGA (71\% versus $45 \% ; p<0.05)$ and the Fried criteria $(58 \%$ versus $14 \% ; p<0.00$ ), to be older of age (mean 83.7 years versus 80.24 years; $p<0.05$ ), and to have a lower BMI at diagnosis (25.12 versus $27.92, p<0.05)$.

Table 3 shows statistically significant differences in sarcopenia, age at diagnosis, sex, low SMI, Fried criteria, and GFI between patients with and without frailty, diagnosed with CGA. Frail patients were more likely to be sarcopenic (44\% versus $21 \%, p<0.05$ ), to be older of age (mean 83.5 years versus 79.0 years; $p<0.05$ ), to be female ( $69 \%$ versus $38 \%$, $p<0.05$ ), to have a low SMI at diagnosis ( $90 \%$ versus $68 \%$, $p<0.05$ ), to be frail according to the Fried criteria (49\% versus 6\%; $p<0.00)$, and the GFI (77\% versus $24 \% ; p<0.00)$.

\section{Univariate and multivariate logistic regressions analyses}

Table 4 shows the univariate and multivariate logistic regression analyses with sarcopenia as the dependent variable. The univariate regression analysis with sarcopenia as dependent variables distinguished age at diagnosis (OR $3.39,95 \%$ CI $1.51-9.99, p=0.027$ ), BMI (OR 0.87, 95\% CI $0,78-0,98, p=0.024$ ), frailty according CGA (OR 2.98, 95\% CI 1.05-8.47, $p=0.040$ ), and frailty according Fried criteria (OR $1.9295 \%$ CI $1.28-2.87, p=0.002$ ) as significant variables for predicting sarcopenia. These significant variables were subjected to two different multivariate analyses: the first with frailty CGA, and the second with frailty Fried criteria because of assumed multicollinearity. In the first multivariate analysis, only BMI (OR 0.87, 95\% CI $0.77-0.98, p=0.022)$ remained significant. In the second age at diagnosis (OR 3.59, 95\% CI 1.02-12.58, $p=0.046$ ), frailty Fried criteria (OR 1.89, 95\% CI 1.22-2.93, $p=0.004$ ), and BMI (OR 0.87, 95\% CI 0.76-0.99, $p=0.033$ ) remained significant.

Table 5 shows the univariate and multivariate logistic regression analyses with frailty, based on CGA, as the dependent variable. The univariate regression analysis with frailty as dependent variables distinguished CCI (OR $1.3595 \%$ CI 1,03-1,76, $p=0.029$ ), HSG (OR 0.92, 95\% CI $0.87-0.97, p=0.006$ ), SMI (OR $0.89,95 \%$ CI $0.83-0.96$, $p=0.002$ ), and sarcopenia (OR $2.98,95 \%$ CI $1.05-8.47$, $p=0.040$ ) as significant variables for predicting frailty. These significant variables were subjected to a multivariate analysis. The first with sarcopenia and the second with HSG and SMI because of assumed multicollinearity. In the second only, SMI (OR 0.89, 95\% CI 0.82-0.96, $p=0.003$ ) remained significant.

\section{Discussion}

In this study, the association between sarcopenia and frailty in 73 participants was retrospectively examined. Sarcopenia is associated with frailty defined with the CGA and Fried criteria, but not with the GFI frailty screening. Furthermore, the Fried criteria and BMI are significant predictors for sarcopenia. Frailty based on the CGA shows associations with the SMI and sarcopenia. Moreover, SMI shows to be a reliable predictor for frailty based on CGA. To our knowledge, this is the first study that examined the association between sarcopenia, as defined by low MF and low SMI, and frailty, as determined by CGA, in HNC patients.

With the aging of the global population, the incidence of frail and sarcopenic patients with HNC will increase. Understandings of the underlying interrelationship of sarcopenia and frailty are of great importance as they are both associated with adverse health outcome [7, 28]. Frailty and sarcopenia are important concepts in preventing physical dependence, as geriatrics are shifting towards identification of early stage of disability. Definitions of both sarcopenia and frailty are still developing, and both concepts clearly overlap in their physical aspects $[11,29]$. Frailty is a pre-disability syndrome where an older person can be identified as being at risk when exposed to stressors associated with high risk for disability or needing to be hospitalized [30]. Two major frailty definitions exist. The physical phenotype of frailty (Fried) [26] and the multiple deficit model (Rockwood) [31]. CGA is the most appropriate way to detect frailty. Frailty is predisposed by advancing age in combination with physiological deterioration, especially a loss of muscle mass. So, sarcopenia is a major driver of frailty, because of decline of MF with low SMM. This increases the risk of falls, which can lead to loss of independence and disability. And low SMM increases the risk of comorbidity like diabetes mellitus and cardiovascular diseases by changing the body fat composition [30].

Studies using "physical" frailty as definition in examining the interrelationship with sarcopenia are suggested to have more overlap [29]. In this study, sarcopenic patients 
Table 2 Characteristics of patients with and without sarcopenia

\begin{tabular}{|c|c|c|c|c|c|c|c|c|}
\hline \multirow[b]{3}{*}{ Age (years) (M, SD) } & \multirow{2}{*}{\multicolumn{2}{|c|}{$\frac{\text { Total }}{N=73}$}} & \multirow{2}{*}{\multicolumn{2}{|c|}{$\begin{array}{l}\text { Sarcopenic } \\
N=24\end{array}$}} & \multirow{2}{*}{\multicolumn{2}{|c|}{$\begin{array}{l}\text { Non sarcopenic } \\
N=49\end{array}$}} & \multirow{3}{*}{$\frac{\chi^{2}}{\text { NA }}$} & \multirow{3}{*}{$\begin{array}{c}p \text { value } \\
0.025\end{array}$} \\
\hline & & & & & & & & \\
\hline & 81.73 & $6.24(\mathrm{SD})$ & 83.7 & $5.73(\mathrm{SD})$ & 80.24 & $6.21(\mathrm{SD})$ & & \\
\hline \multicolumn{9}{|l|}{$\operatorname{Sex}(n, \%)$} \\
\hline Male & 33 & 45 & 10 & 42 & 23 & 47 & \multirow[t]{2}{*}{0.181} & \multirow[t]{2}{*}{0.671} \\
\hline Female & 40 & 55 & 14 & 58 & 26 & 53 & & \\
\hline \multicolumn{9}{|c|}{ Weight loss 6 months prior to diagnosis (n, \%) } \\
\hline Non & 46 & 63 & 15 & 63 & 31 & 63 & \multirow[t]{3}{*}{0.392} & \multirow[t]{3}{*}{0.822} \\
\hline$<10 \%$ & 20 & 27 & 6 & 25 & 14 & 29 & & \\
\hline$\geq 10 \%$ & 7 & 10 & 3 & 13 & 4 & 8 & & \\
\hline BMI $\left(\mathrm{kg} / \mathrm{m}^{2}\right)$ & 26.80 & $5.70(\mathrm{SD})$ & 25.12 & 4.99(SD) & 27.92 & 4.49(SD) & NA & 0.018 \\
\hline \multicolumn{9}{|l|}{ Smoker $(n, \%)$} \\
\hline No & 25 & 34 & 11 & 46 & 14 & 29 & \multirow[t]{3}{*}{2.604} & \multirow[t]{3}{*}{0.272} \\
\hline Former & 40 & 55 & 10 & 42 & 30 & 61 & & \\
\hline Current & 8 & 11 & 3 & 13 & 5 & 10 & & \\
\hline \multicolumn{9}{|l|}{$\operatorname{MUST}$ score $(n, \%)$} \\
\hline 0 & 53 & 72 & 18 & 75 & 35 & 71 & \multirow[t]{3}{*}{0.533} & \multirow[t]{3}{*}{0.766} \\
\hline 1 & 1 & 2 & 0 & 0 & 1 & 2 & & \\
\hline 2 & 19 & 26 & 6 & 25 & 13 & 27 & & \\
\hline \multicolumn{9}{|l|}{ Alcohol use $(n, \%)$} \\
\hline No & 25 & 34 & 9 & 38 & 16 & 33 & 1.340 & 0.720 \\
\hline Yes & 41 & 56 & 14 & 58 & 27 & 55 & & \\
\hline Former & 7 & 10 & 1 & 4 & 6 & 12 & & \\
\hline Charlson comorbidity & index $(r$ & & & & & & & \\
\hline Low $\leq 6$ & 27 & 37 & 6 & 25 & 21 & 43 & 2.204 & 0.138 \\
\hline High $>6$ & 46 & 63 & 18 & 75 & 28 & 57 & & \\
\hline Localization $(n, \%)$ & & & & & & & & \\
\hline Oral cavity & 46 & 63 & 14 & 58 & 32 & 65 & 12.284 & 0.584 \\
\hline Nasopharynx & 2 & 3 & 1 & 4 & 1 & 2 & & \\
\hline Oropharynx & 2 & 3 & 0 & 0 & 2 & 4 & & \\
\hline Hypopharynx & 3 & 4 & 1 & 4 & 2 & 4 & & \\
\hline Larynx & 7 & 10 & 2 & 8 & 5 & 10 & & \\
\hline Skin & 8 & 11 & 3 & 13 & 5 & 10 & & \\
\hline Paranasal sinuses & 2 & 2 & 1 & 4 & 2 & 4 & & \\
\hline Type of tumor $(n, \%)$ & & & & & & & & \\
\hline Primary & 56 & 77 & 17 & 71 & 39 & 80 & 1.019 & 0.601 \\
\hline Recurrent & 11 & 15 & 4 & 17 & 7 & 14 & & \\
\hline Second primary & 6 & 8 & 3 & 13 & 3 & 6 & & \\
\hline TNM Stage $(n, \%)$ & & & & & & & & \\
\hline I & 8 & 11 & 5 & 21 & 3 & 6 & 3.708 & 0.295 \\
\hline II & 19 & 26 & 5 & 21 & 14 & 29 & & \\
\hline III & 14 & 19 & 4 & 17 & 10 & 20 & & \\
\hline IV & 32 & 44 & 10 & 42 & 22 & 45 & & \\
\hline Low Muscle strength & $n, \%)$ & & & & & & & \\
\hline No & 40 & 55 & 0 & 0 & 40 & 82 & 43.340 & 0.000 \\
\hline Yes & 33 & 45 & 24 & 100 & 9 & 18 & & \\
\hline Low SMI $(n, \%)$ & & & & & & & & \\
\hline No & 15 & 21 & 0 & 0 & 15 & 31 & 9.247 & 0.002 \\
\hline Yes & 58 & 79 & 24 & 100 & 34 & 69 & & \\
\hline Frailty Fried criteria & $n, \%)$ & & & & & & & \\
\hline No & 52 & 71 & 10 & 42 & 42 & 86 & 15.253 & 0.000 \\
\hline
\end{tabular}


Table 2 (continued)

\begin{tabular}{|c|c|c|c|c|c|c|c|c|}
\hline \multirow[b]{3}{*}{ Yes } & \multirow{2}{*}{\multicolumn{2}{|c|}{$\frac{\text { Total }}{N=73}$}} & \multirow{2}{*}{\multicolumn{2}{|c|}{$\begin{array}{l}\text { Sarcopenic } \\
N=24\end{array}$}} & \multirow{2}{*}{\multicolumn{2}{|c|}{$\begin{array}{l}\text { Non sarcopenic } \\
N=49\end{array}$}} & \multirow[t]{3}{*}{$\chi^{2}$} & \multirow[t]{3}{*}{$p$ value } \\
\hline & & & & & & & & \\
\hline & 21 & 29 & 14 & 58 & 7 & 14 & & \\
\hline \multicolumn{9}{|c|}{ Frailty GFI $(n, \%)$} \\
\hline No & 35 & 50 & 9 & 38 & 26 & 53 & \multirow[t]{2}{*}{1.563} & \multirow[t]{2}{*}{0.211} \\
\hline Yes & 38 & 52 & 15 & 63 & 23 & 47 & & \\
\hline \multicolumn{9}{|c|}{ Frailty CGA $(n, \%)$} \\
\hline No & 34 & 46 & 7 & 29 & 27 & 55 & \multirow[t]{2}{*}{4.355} & \multirow[t]{2}{*}{0.037} \\
\hline Yes & 39 & 54 & 17 & 71 & 22 & 45 & & \\
\hline
\end{tabular}

$B M$ body mass index, $M U S T$ malnutrition universal screening tool, $S M I$ skeletal muscle index, GFI groningen frailty indicator, $C G A$ comprehensive geriatric assessment

were more likely to be frail, according to the Fried criteria. Moreover, the Fried criterion was an independent predictor for sarcopenia. GFI was not associated with sarcopenia. Presumably because GFI uses also social, and psychological domains rather than only physically items like the Fried criteria. This confirms that "physical" frailty, like the Fried criteria, is more associated with sarcopenia than definitions based upon the multiple deficit model (Rockwood).

A previous retrospective study found a significant association between sarcopenia and frailty based on the G8 questionnaire (OR 0.76, 95\% CI 0.6-0.89, $p<0.001)$ [10]. In that study, sarcopenia was based only on low SMI, so according to the EWGSOP-2 criterion, it was insufficient as sarcopenia which includes muscle function as well. Also, frailty screening was based on different screening questionnaires, i.e. G8, Timed Up and Go test, and Malnutrition Universal Screening Tool. In our study, SMI, but not the combination of low MF and low SMI (defined as sarcopenia by the EWGSOP-2), was independently associated with frailty based on CGA (OR 0.89, 95\% CI 0.82-0.96, $p=0.003$ ).

The suggestion that SMI could possibly be able to predict frailty, in particularly the physical part of frailty, in patients with $\mathrm{HNC}$ and is easier to use and implement, then a CGA or questionnaires to diagnose frailty is in accordance with the study of Zwart et al., although in our study, SMI was directly associated with CGA instead of the G8 frailty screening questionnaire [10].

Our study has some limitations. It was designed as a retrospective single-center study, with a limited number of included patients. Only patients with the available data on MF and SMI were included in the study. As it is more likely that MF parameters were examined for frail patients than for fit patients, this may have resulted in selection bias. Also, both CT and MRI imaging are used for the assessment of SMI, to maximize the number of patients that could be included. This could raise concerns but these two different imaging modalities show significant correlation in quantifying SMI when measured by CSA at the level of C3 [32]. At last, the majority (63\%) of the patients had an oral cavity cancer. Other tumor locations as pharynx may cause more significant weight loss prior to diagnosis. In the present study, we used the commercially available software package SliceOmatic, but also free open-source software applications, e.g., 3D Slicer, can be used to assess CSA.

A strength of our study is that all of the muscle tissues were manually performed by a single researcher who was blinded for outcome regarding frailty and sarcopenia. Because an excellent inter-observer agreement for SMI measurement at the level of $\mathrm{C} 3$ was demonstrated, these SMI measurement findings can be used globally to tailor treatment according patients' frailty [33].

In conclusion, there is an association between sarcopenia and frailty defined by CGA. Low muscle mass, based on SMI, may be able to predict some CGA domain outcomes in older patients with $\mathrm{HNC}$ and is easier to use and implement then a CGA. These findings should ideally be validated in a larger, prospective cohort study. 
Table 3 Characteristics of patients with and without frailty based on the CGA

\begin{tabular}{|c|c|c|c|c|c|c|c|c|}
\hline \multirow[b]{3}{*}{ Age (years) (M, SD) } & \multirow{2}{*}{\multicolumn{2}{|c|}{$\frac{\text { Total }}{N=73}$}} & \multirow{2}{*}{\multicolumn{2}{|c|}{$\frac{\text { Frail }}{N=39}$}} & \multirow{2}{*}{\multicolumn{2}{|c|}{$\begin{array}{l}\text { Non frail } \\
N=34\end{array}$}} & \multirow{3}{*}{$\begin{aligned} \chi^{2} \\
\mathrm{NA}\end{aligned}$} & \multirow{3}{*}{$\begin{array}{l}p \text { value } \\
\mathbf{0 . 0 0 2}\end{array}$} \\
\hline & & & & & & & & \\
\hline & 81.73 & $6.24(\mathrm{SD})$ & 83.49 & $6.47(\mathrm{SD})$ & 78.96 & $5.03(\mathrm{SD})$ & & \\
\hline \multicolumn{9}{|l|}{$\operatorname{Sex}(n, \%)$} \\
\hline Male & 33 & 45 & 12 & 31 & 21 & 62 & \multirow[t]{2}{*}{7.045} & \multirow[t]{2}{*}{0.008} \\
\hline Female & 40 & 55 & 27 & 69 & 13 & 38 & & \\
\hline \multicolumn{9}{|c|}{ Weight loss 6 months prior to diagnosis $(n, \%)$} \\
\hline Non & 46 & 63 & 22 & 56 & 24 & 71 & \multirow[t]{3}{*}{1.839} & \multirow[t]{3}{*}{0.399} \\
\hline$<10 \%$ & 20 & 27 & 12 & 31 & 8 & 24 & & \\
\hline$\geq 10 \%$ & 7 & 10 & 5 & 13 & 2 & 6 & & \\
\hline BMI (kg/m2) & 26.80 & $5.70(\mathrm{SD})$ & 26.98 & $5.66(\mathrm{SD})$ & 27.02 & $3.69(\mathrm{SD})$ & NA & 0.970 \\
\hline \multicolumn{9}{|l|}{ Smoker $(n, \%)$} \\
\hline No & 25 & 34 & 15 & 38 & 10 & 29 & \multirow[t]{3}{*}{3.072} & \multirow[t]{3}{*}{0.215} \\
\hline Former & 40 & 55 & 18 & 46 & 22 & 65 & & \\
\hline Current & 8 & 11 & 6 & 15 & 2 & 6 & & \\
\hline \multicolumn{9}{|l|}{ MUST score $(n, \%)$} \\
\hline 0 & 53 & 72 & 24 & 62 & 29 & 85 & \multirow[t]{3}{*}{7.533} & \multirow[t]{3}{*}{0.023} \\
\hline 1 & 1 & 2 & 0 & 0 & 1 & 3 & & \\
\hline 2 & 19 & 26 & 15 & 38 & 4 & 12 & & \\
\hline \multicolumn{9}{|l|}{ Alcohol use $(n, \%)$} \\
\hline No & 25 & 34 & 15 & 38 & 10 & 29 & 1.498 & 0.683 \\
\hline Yes & 41 & 56 & 21 & 54 & 20 & 59 & & \\
\hline Former & 7 & 10 & 3 & 8 & 4 & 12 & & \\
\hline Charlson comorbidity & index $(r$ & & & & & & & \\
\hline Low $\leq 6$ & 27 & 37 & 10 & 26 & 17 & 50 & 4.624 & 0.032 \\
\hline High $>6$ & 46 & 63 & 29 & 74 & 17 & 50 & & \\
\hline Localization $(n, \%)$ & & & & & & & & \\
\hline Oral cavity & 46 & 63 & 27 & 69 & 19 & 56 & 17.392 & 0.236 \\
\hline Nasopharynx & 2 & 3 & 1 & 3 & 1 & 3 & & \\
\hline Oropharynx & 2 & 3 & 1 & 3 & 1 & 3 & & \\
\hline Hypopharynx & 3 & 4 & 0 & 0 & 3 & 9 & & \\
\hline Larynx & 7 & 10 & 2 & 5 & 5 & 15 & & \\
\hline Salivary glands & 3 & 4 & 2 & 5 & 1 & 3 & & \\
\hline Skin & 8 & 11 & 5 & 13 & 3 & 9 & & \\
\hline Paranasal sinuses & 2 & 2 & 1 & 3 & 1 & 3 & & \\
\hline Type of tumor $(n, \%)$ & & & & & & & & \\
\hline Primary & 56 & 77 & 28 & 72 & 28 & 82 & 5.776 & 0.056 \\
\hline Recurrent & 11 & 15 & 5 & 13 & 6 & 18 & & \\
\hline Second primary & 6 & 8 & 6 & 15 & 0 & 0 & & \\
\hline TNM Stage $(n, \%)$ & & & & & & & & \\
\hline I & 8 & 11 & 5 & 13 & 3 & 9 & 1.047 & 0.790 \\
\hline II & 19 & 26 & 11 & 28 & 8 & 24 & & \\
\hline III & 14 & 19 & 6 & 15 & 8 & 24 & & \\
\hline IV & 32 & 44 & 17 & 44 & 15 & 44 & & \\
\hline Low muscle strength & $n, \%)$ & & & & & & & \\
\hline No & 40 & 55 & 20 & 51 & 20 & 59 & 0.417 & 0.518 \\
\hline Yes & 33 & 45 & 19 & 49 & 14 & 41 & & \\
\hline No & 15 & 21 & 4 & 10 & 11 & 32 & 5.432 & 0.020 \\
\hline Yes & 58 & 79 & 35 & 90 & 23 & 68 & & \\
\hline Frailty fried criteria ( & $\%)$ & & & & & & & \\
\hline No & 52 & 71 & 20 & 51 & 32 & 94 & 16.265 & 0.000 \\
\hline
\end{tabular}


Table 3 (continued)

\begin{tabular}{|c|c|c|c|c|c|c|c|c|}
\hline \multirow[b]{3}{*}{ Yes } & \multirow{2}{*}{\multicolumn{2}{|c|}{$\frac{\text { Total }}{N=73}$}} & \multirow{2}{*}{\multicolumn{2}{|c|}{$\begin{array}{l}\text { Frail } \\
N=39\end{array}$}} & \multirow{2}{*}{\multicolumn{2}{|c|}{$\begin{array}{l}\text { Non frail } \\
N=34\end{array}$}} & \multirow[t]{3}{*}{$\chi^{2}$} & \multirow[t]{3}{*}{$p$ value } \\
\hline & & & & & & & & \\
\hline & 21 & 29 & 19 & 49 & 2 & 6 & & \\
\hline \multicolumn{9}{|c|}{ Frailty GFI $(n, \%)$} \\
\hline No & 35 & 50 & 9 & 23 & 26 & 76 & \multirow[t]{2}{*}{20.749} & \multirow[t]{2}{*}{0.000} \\
\hline Yes & 38 & 52 & 30 & 77 & 8 & 24 & & \\
\hline \multicolumn{9}{|c|}{ Sarcopenia $(n, \%)$} \\
\hline No & 49 & 67 & 22 & 56 & 27 & 79 & \multirow[t]{2}{*}{4.355} & \multirow[t]{2}{*}{$\mathbf{0 . 0 3 7}$} \\
\hline Yes & 24 & 33 & 17 & 44 & 7 & 21 & & \\
\hline
\end{tabular}

BMI Body mass index, MUST Malnutrition universal screening tool, SMI skeletal muscle index, GFI groningen frailty indicator, $C G A$ comprehensive geriatric assessment

Table 4 Univariate and multivariate logistic regression analysis for analyzing variables associated with sarcopenia in HNC patients

\begin{tabular}{|c|c|c|c|c|c|c|c|c|c|}
\hline \multirow[t]{2}{*}{ Sarcopenia } & \multicolumn{3}{|c|}{ Univariate analysis } & \multicolumn{6}{|c|}{ Multivariate analysis } \\
\hline & OR & $95 \% \mathrm{CI}$ & $p$ value & OR & $95 \% \mathrm{CI}$ & $p$ value & OR & $95 \% \mathrm{CI}$ & $p$ value \\
\hline \multicolumn{10}{|l|}{ Age (years) } \\
\hline$\leq 80$ & Ref & & & Ref & & & Ref & & \\
\hline$>80$ & 3.391 & $1.51-9.99$ & $0.027 * *$ & 3.196 & $0.98-10.34$ & 0.052 & 3.587 & $1.02-12.58$ & $0.046 * *$ \\
\hline \multicolumn{10}{|l|}{ Sex } \\
\hline Male & Ref & & & & & & & & \\
\hline Female & 1.238 & $0.46-3.32$ & 0.671 & & & & & & \\
\hline \multicolumn{10}{|c|}{ Weight loss 6 months prior to diagnosis } \\
\hline Non & Ref & & & & & & & & \\
\hline$<10 \%$ & 0.886 & $0.28-2.76$ & 0.834 & & & & & & \\
\hline$\geq 10 \%$ & 1.550 & $0.31-7.82$ & 0.596 & & & & & & \\
\hline BMI $(\mathrm{kg} / \mathrm{m} 2)$ & 0.873 & $0.78-0.98$ & $0.024 * *$ & 0.867 & $0.77-0.98$ & $\mathbf{0 . 0 2 2} * *$ & 0.869 & $0.76-0.99$ & $\mathbf{0 . 0 3 3} * *$ \\
\hline Charlson comorbidity index & 1.299 & $0.99-1.70$ & 0.058 & & & & & & \\
\hline \multicolumn{10}{|l|}{ TNM Stage } \\
\hline I & Ref & & & & & & & & \\
\hline II & 0.214 & $0.03-1.24$ & 0.086 & & & & & & \\
\hline III & 0.240 & $0.04-1.51$ & 0.129 & & & & & & \\
\hline IV & 0.273 & $0.05-1.37$ & 0.115 & & & & & & \\
\hline Frailty Fried criteria & 1.916 & $1.28-2.87$ & $0.002 *$ & & & & 1.892 & $1.22-2.93$ & $0.004 *$ \\
\hline \multicolumn{10}{|l|}{ Frailty GFI } \\
\hline No & Ref & & & & & & & & \\
\hline Yes & 1.884 & $0.69-5.12$ & 0.214 & & & & & & \\
\hline \multicolumn{10}{|l|}{ Frailty CGA } \\
\hline No & Ref & & & Ref & & & & & \\
\hline Yes & 2.981 & $1.05-8.47$ & $0.040 * *$ & 2.537 & $0.83-7.76$ & 0.103 & & & \\
\hline
\end{tabular}

The first with multivariate analysis is conducted with Frailty CGA and the second with Frailty Fried criteria because of assumed multicollinearity

$B M I$ body mass index, GFI groningen grailty indicator, $C G A$ comprehensive geriatric assessment

${ }^{*}$ Correlation is significant at the 0.01 level (2-tailed)

${ }^{* *}$ Correlation is significant at the 0.05 level (2-tailed) 
Table 5 Univariate and multivariate logistic regression analysis for analyzing variables associated with frailty based on CGA in HNC patients

\begin{tabular}{|c|c|c|c|c|c|c|c|c|c|}
\hline \multirow[t]{2}{*}{ Frailty } & \multicolumn{3}{|c|}{ Univariate analysis } & \multicolumn{6}{|c|}{ Multivariate analysis } \\
\hline & OR & $95 \%$ CI & $p$ value & OR & $95 \% \mathrm{CI}$ & $p$ value & OR & $95 \%$ CI & $p$ value \\
\hline \multicolumn{10}{|l|}{ Age (years) } \\
\hline$\leq 80$ & Ref & & & & & & & & \\
\hline$>80$ & 2.533 & $0.98-6.55$ & 0.055 & & & & & & \\
\hline $\begin{array}{l}\text { Charlson } \\
\text { comorbidity } \\
\text { index }\end{array}$ & 1.350 & $1.03-1.76$ & $0.029 * *$ & 1.294 & $0.98-1.69$ & 0.064 & 1.328 & $0.99-1.79$ & 0.061 \\
\hline \multicolumn{10}{|l|}{ TNM Stage } \\
\hline I & Ref & & & & & & & & \\
\hline II & 0.825 & $0.15-4.50$ & 0.824 & & & & & & \\
\hline III & 0.450 & $0.08-2.67$ & 0.379 & & & & & & \\
\hline IV & 0.680 & $0.14-3.34$ & 0.635 & & & & & & \\
\hline HSG & 0.922 & $0.87-0.97$ & $0.006 *$ & & & & 0.941 & $0.82-1.00$ & 0.060 \\
\hline SMI & 0.893 & $0.83-0.96$ & $0.002 *$ & & & & 0.887 & $0.82-0.96$ & 0.003* \\
\hline \multicolumn{10}{|l|}{ Sarcopenia } \\
\hline No & Ref & & & & & & & & \\
\hline Yes & 2.981 & $1.05-8.47$ & $0.040 * *$ & 2.494 & $0.85-7.34$ & 0.097 & & & \\
\hline
\end{tabular}

The first with multivariate analysis is conducted with Frailty CGA and the second with Frailty Fried criteria because of assumed multicollinearity

$B M I$ body mass indexm, GFI groningen frailty indicator, CGA comprehensive geriatric assessment

${ }^{*}$ Correlation is significant at the 0.01 level (2-tailed)

${ }^{* *}$ Correlation is significant at the 0.05 level (2-tailed)
Open Access This article is licensed under a Creative Commons Attribution 4.0 International License, which permits use, sharing, adaptation, distribution and reproduction in any medium or format, as long as you give appropriate credit to the original author(s) and the source, provide a link to the Creative Commons licence, and indicate if changes were made. The images or other third party material in this article are included in the article's Creative Commons licence, unless indicated otherwise in a credit line to the material. If material is not included in the article's Creative Commons licence and your intended use is not permitted by statutory regulation or exceeds the permitted use, you will need to obtain permission directly from the copyright holder. To view a copy of this licence, visit http://creativecommons.org/licenses/by/4.0/.

\section{References}

1. Bray F, Ferlay J, Soerjomataram I et al (2018) Global cancer statistics 2018: GLOBOCAN estimates of incidence and mortality worldwide for 36 cancers in 185 countries. CA Cancer J Clin 68:394-424. https://doi.org/10.3322/caac.21492

2. Stoyanov GS, Kitanova M, Dzhenkov DL et al (2017) Demographics of head and neck cancer patients: a single institution experience. Cureus 9:e1418. https://doi.org/10.7759/cureus.1418

3. Boje CR (2014) Impact of comorbidity on treatment outcome in head and neck squamous cell carcinoma-a systematic review. Radiother Oncol 110:81-90. https://doi.org/10.1016/j.radonc. 2013.07.005

4. Argiris A, Karamouzis MV, Raben D, Ferris RL (2008) Head and neck cancer. Lancet (London, England) 371:1695-1709. https:// doi.org/10.1016/S0140-6736(08)60728-X
5. Mehanna H, West CML, Nutting C, Paleri V (2010) Head and neck cancer-Part 2: treatment and prognostic factors. BMJ 341:c4690. https://doi.org/10.1136/bmj.c4690

6. Langendijk JA, Doornaert P, Verdonck-de Leeuw IM et al (2008) Impact of late treatment-related toxicity on quality of life among patients with head and neck cancer treated with radiotherapy. J Clin Oncol 26:3770-3776. https://doi.org/10.1200/JCO.2007.14. 6647

7. Pottel L, Lycke M, Boterberg T et al (2014) Serial comprehensive geriatric assessment in elderly head and neck cancer patients undergoing curative radiotherapy identifies evolution of multidimensional health problems and is indicative of quality of life. Eur J Cancer Care (Engl) 23:401-412. https://doi.org/10.1111/ ecc. 12179

8. Extermann M, Aapro M, Bernabei R et al (2005) Use of comprehensive geriatric assessment in older cancer patients: recommendations from the task force on CGA of the International Society of Geriatric Oncology (SIOG). Crit Rev Oncol Hematol 55:241-252. https://doi.org/10.1016/j.critrevonc.2005.06.003

9. Hamaker ME, Jonker JM, de Rooij SE et al (2012) Frailty screening methods for predicting outcome of a comprehensive geriatric assessment in elderly patients with cancer: a systematic review. Lancet Oncol 13:e437-e444. https://doi.org/10.1016/ S1470-2045(12)70259-0

10. Zwart AT, van der Hoorn A, van Ooijen PMA et al (2019) CTmeasured skeletal muscle mass used to assess frailty in patients with head and neck cancer. J Cachexia Sarcopenia Muscle 10:1060-1069. https://doi.org/10.1002/jcsm.12443

11. Reijnierse EM, Trappenburg MC, Blauw GJ et al (2016) Common ground? the concordance of sarcopenia and frailty definitions. J Am Med Dir Assoc 17:371.e7-12. https://doi.org/10. 1016/j.jamda.2016.01.013 
12. Cruz-Jentoft AJ, Bahat G, Bauer J et al (2019) Sarcopenia: revised European consensus on definition and diagnosis. Age Ageing 48:16-31. https://doi.org/10.1093/ageing/afy 169

13. Wendrich AW, Swartz JE, Bril SI et al (2017) Low skeletal muscle mass is a predictive factor for chemotherapy dose-limiting toxicity in patients with locally advanced head and neck cancer. Oral Oncol 71:26-33. https://doi.org/10.1016/j.oraloncology. 2017.05.012

14. Achim V, Bash J, Mowery A et al (2017) Prognostic indication of sarcopenia for wound complication after total laryngectomy. JAMA Otolaryngol Head Neck Surg 143:1159-1165. https:// doi.org/10.1001/jamaoto.2017.0547

15. Nishikawa D, Hanai N, Suzuki H et al (2018) The impact of skeletal muscle depletion on head and neck squamous cell carcinoma. ORL J Otorhinolaryngol Relat Spec 80:1-9. https://doi. org/10.1159/000485515

16. Lauretani F, Russo CR, Bandinelli S et al (2003) Age-associated changes in skeletal muscles and their effect on mobility: an operational diagnosis of sarcopenia. J Appl Physiol 95:1851-1860. https://doi.org/10.1152/japplphysiol.00246.2003

17. Heymsfield SB, Wang Z, Baumgartner RN, Ross R (1997) Human body composition: advances in models and methods. Annu Rev Nutr 17:527-558. https://doi.org/10.1146/annurev.nutr.17.1.527

18. Swartz JE, Pothen AJ, Wegner I et al (2016) Feasibility of using head and neck CT imaging to assess skeletal muscle mass in head and neck cancer patients. Oral Oncol 62:28-33. https://doi.org/10. 1016/j.oraloncology.2016.09.006

19. Charlson ME, Pompei P, Ales KL, MacKenzie CR (1987) A new method of classifying prognostic comorbidity in longitudinal studies: development and validation. J Chronic Dis 40:373-383. https://doi.org/10.1016/0021-9681(87)90171-8

20. Elia M (2013) The "MUST" report. Nutritional screening for adults: a multidisciplinary responsibility. Development and use of the "Malnutrition Universal Screening Tool" (MUST) for adults

21. Folstein MF, Folstein SE, McHugh PR (1975) "Mini-mental state". A practical method for grading the cognitive state of patients for the clinician. J Psychiatr Res 12:189-198. https://doi. org/10.1016/0022-3956(75)90026-6

22. Yesavage JA, Sheikh JI (1986) $9 /$ Geriatric depression scale (GDS). Clin Gerontol 5:165-173. https://doi.org/10.1300/J018v 05n01_09

23. Katz S, Ford AB, Moskowitz RW, Jackson BA, Jaffe MW (1963) Studies of illness in the aged. The index of ADL: a standardized measure of biological and psychosocial function. JAMA 185:914 919. https://doi.org/10.1001/jama.1963.03060120024016
24. Weinberger M, Samsa GP, Schmader K et al (1992) Comparing proxy and patients' perceptions of patients' functional status: results from an outpatient geriatric clinic. J Am Geriatr Soc 40:585-588. https://doi.org/10.1111/j.1532-5415.1992.tb02107.x

25. Fried LP, Tangen CM, Walston J et al (2001) Frailty in older adults: evidence for a phenotype. J Gerontol A Biol Sci Med Sci 56:M146-M156. https://doi.org/10.1093/gerona/56.3.m146

26. Goldstein DP, Sklar MC, de Almeida JR et al (2020) Frailty as a predictor of outcomes in patients undergoing head and neck cancer surgery. Laryngoscope 130:E340-E345. https://doi.org/10. 1002/lary.28222

27. Soubeyran P, Bellera CA, Gregoire F et al (2008) Validation of a screening test for elderly patients in oncology. J Clin Oncol 26:20568. https://doi.org/10.1200/jco.2008.26.15_suppl.20568

28. Chargi N, Bril SI, Emmelot-Vonk MH, de Bree R (2019) Sarcopenia is a prognostic factor for overall survival in elderly patients with head-and-neck cancer. Eur Arch Oto-Rhino-Laryngology 276:1475-1486. https://doi.org/10.1007/s00405-019-05361-4

29. Mijnarends DM, Schols JMGA, Meijers JMM et al (2015) Instruments to assess sarcopenia and physical frailty in older people living in a community (care) setting: similarities and discrepancies. J Am Med Dir Assoc 16:301-308. https://doi.org/10.1016/j.jamda. 2014.11.011

30. Pel-Littel RE, Schuurmans MJ, Emmelot-Vonk MH, Verhaar HJJ (2009) Frailty: defining and measuring of a concept. JNHA - J Nutr Heal Aging 13:390-394. https://doi.org/10.1007/ s12603-009-0051-8

31. Rockwood K, Stadnyk K, MacKnight C et al (1999) A brief clinical instrument to classify frailty in elderly people. Lancet (London, England) 353:205-206

32. Chargi N, Ansari E, Huiskamp LFJ et al (2019) Agreement between skeletal muscle mass measurements using computed tomography imaging and magnetic resonance imaging in head and neck cancer patients. Oral Oncol 99:104341

33. Bril SI, Wendrich AW, Swartz JE et al (2019) Interobserver agreement of skeletal muscle mass measurement on head and neck CT imaging at the level of the third cervical vertebra. Eur Arch Otorhinolaryngol 276:1175-1182. https://doi.org/10.1007/ s00405-019-05307-w

Publisher's Note Springer Nature remains neutral with regard to jurisdictional claims in published maps and institutional affiliations. 